\title{
Health-Related Quality of Life, Activities of Daily Living and Parenting Stress in Children with Brain Tumors
}

\author{
Kyung Jin An¹, Mi Sun Song ${ }^{1}$, Ki Woong Sung ${ }^{2}$ and Yoo Sook Joung ${ }^{\bowtie}$ \\ ${ }^{1}$ Departments of Psychiatry and ${ }^{2}$ Pediatrics, Samsung Medical Center, Sungkyunkwan University School of Medicine, Seoul, Korea
}

\begin{abstract}
Objective To compare health-related quality of life (HRQOL), activities of daily living (ADL), and parenting stress between children with brain tumors and those of normal control (NC).

Methods Participants were 31 brain tumor patients who had received chemotherapy, radiotherapy, or peripheral blood stem cell transplantation after surgical resection and $125 \mathrm{NC}$ subjects. We administered the Pediatric Quality of Life Inventory, version 4.0 (PedsQL), to assess HRQOL; the Korean version of the Parenting Stress Index-Short Form (K-PSI-SF), to assess parenting stress; and examined ADL.

Results The mean self-report PedsQL scores for physical health, emotional functioning, social functioning, school functioning, and total scores in the patients were significantly lower than those of the NC group. The mean parent proxy-report PedsQL scores for all scales except emotional functioning in the patient group were significantly lower than those of the NC group. The ADL impairment for the patients was significantly more than that of the NC group. The K-PSI-SF mean scores for stress related to having a difficult child and for stress related to parent-child interaction in the patient group tended to be higher than those of the NC group ( $\mathrm{p}=0.09)$.

Conclusion The children with brain tumors had lower HRQOL and difficulties in performing ADL. The parents of children with brain tumors tended to experience stress related to having a difficult child and to parent-child interaction. We suggest that long-term monitors for the child's HRQOL and ADL, and parenting stress in children with brain tumors are needed.

Psychiatry Investig 2011;8:250-255
\end{abstract}

Key Words ADL, Childhood brain tumor, HRQOL, Parenting stress.

\section{INTRODUCTION}

Brain tumors are the second most common form of childhood cancer, accounting for approximately $20 \%$ of childhood cancer diagnoses. ${ }^{1}$ Survival rates have increased markedly over the last several decades due to aggressive combinations of therapies, including surgical resection, chemotherapy, radiotherapy, and peripheral blood stem cell transplantation. Nowadays, approximately $65 \%$ of all children treated for brain tumors achieve long-term survival. ${ }^{2}$ Therefore the perspective of brain tumor as a chronic disease has appeared. Survivors of childhood brain tumors may suffer from persistent psychosocial, behavioral, and social difficulties. Also, caregivers of children who suffered from brain tumors may experience signifi-

Received: December 31, 2010 Revised: April 12, 2011

Accepted: May 3, 2011 Available online: August 2, 2011

$\triangle$ Correspondence: Yoo Sook Joung, $\mathrm{MD}, \mathrm{PhD}$

Department of Psychiatry, Samsung Medical Center, Sungkyunkwan University School of Medicine, 50 Irwon-dong, Gangnam-gu, Seoul 135-710, Korea Tel: +82-2-3410-3589, Fax: +82-2-3410-0050, E-mail: yschoung@skku.edu

(c) This is an Open Access article distributed under the terms of the Creative Commons Attribution Non-Commercial License (http://creativecommons.org/licenses/by$\mathrm{nc} / 3.0$ ) which permits unrestricted non-commercial use, distribution, and reproduction in any medium, provided the original work is properly cited. cant ongoing distress.

Since the treatment, recovery, and rehabilitation of children with brain tumors may be lengthy, such children may have difficulties reintegrating into normal life, maintaining peer relationships, and attaining normal academic milestones. ${ }^{3-5}$ Survivors of childhood brain tumors have reported that they had lower quality of life (QOL) than either their normal peers or other childhood cancer survivors. ${ }^{6-12}$ Also, brain tumor survivors had the highest prevalence of physical limitations. ${ }^{13}$

Caregivers of children with brain tumors reported high levels of distress, post-traumatic stress and lower QOL in both the physical and psychological domains. ${ }^{14,15}$ They reported that stress continued for 5 years or more post-diagnosis. ${ }^{16}$

Pediatric health-related quality of life (HRQOL), which is multidimensional, consisting at the minimum of the physical, psychological (including emotional and cognitive), and social health dimensions delineated by the World Health Organization, ${ }^{17}$ has emerged as an important health outcome in pediatric clinical trials and in strategies to improve clinical outcomes. ${ }^{18,19}$ Therefore, many studies in many countries have considered psychological outcomes, including QOL, in children with brain tumors. A study about children with brain tumors 
in Korea was needed because of trans-cultural and ethnic differences in patients' QOL and stress. ${ }^{20}$ However, few studies have investigated psychological outcomes, including HRQOL, in children with brain tumors in Korea.

We aimed to assess HRQOL, ability to perform Activities of Daily Living (ADL), and parenting stress, satisfaction and efficacy in children with brain tumors and compare these to those of normal controls (NCs). Also, we aimed to assess relationships between clinical characteristics with HRQOL in children with brain tumors.

\section{METHODS}

\section{Subjects}

Study participants were 31 patients aged 3 to 18 years (mean age 9.65 $\pm 2.80,21$ males and 10 females) and $125 \mathrm{NCs}$ (mean age $9.72 \pm 1.51,64$ males and 61 females). The 31 patients had brain tumor diagnoses and received various treatments such as chemotherapy, or radiotherapy, or peripheral blood stem cell transplantation after surgical resection at the Children Cancer Unit at Samsung Medical Center, Seoul, Republic of Korea, from February 2008 to August 2009. When we procedure this cross-sectional study, 15 patients received chemotherapy, 7 patients received chemotherapy and radiotherapy, and 8 patients received chemotherapy, radiotherapy, and peripheral blood stem cell transplantation (Table 1).

\section{Procedure}

For brain tumor patients, we gathered information on diagnosis, treatment stage, size of residual tumor, and presence of leptomeningeal seeding through medical records and used the Korean version of the Wechsler Preschool and Primary Scales of Intelligence (K-WPPSI) ${ }^{21}$ or the Korean version of the Wechsler Intelligence Scale for Children-III (K-WISC-III) ${ }^{22}$ to assess the children's intelligence and the Korean version of the Child Behavior Checklist (K-CBCL) ${ }^{23}$ to assess their behavior problems and social competencies. As CBCL was designed for completion by parents between 4 to 18 years of age, we assessed CBCL for 28 patients older than 4 years.

For both brain tumor patients and NC subjects, we administered the self-report Pediatric Quality of Life Inventory, version 4.0 (PedsQL) ${ }^{24}$ to assess HRQOL and examined ADL such as 1) bowel control; 2) bladder control; 3) washing, brushing, and shampoo; 4) toileting; 5) eating; 6) transferring; 7) ambulation; 8) dressing; 9) stair climbing; 10) bathing; 11) being left alone; and 12) wearing shoes. ${ }^{25}$

The PedsQL measure HRQOLs for the 1-Month period prior to interview, and have 4 domains and 23 items: Physical Functioning (8 items), Emotional Functioning (5 items), Social Functioning (5 items), School Functioning (5 items); and
Table 1. Clinical characteristics of children with brain tumors

\begin{tabular}{lc}
\hline Mean age at diagnosis of brain tumor, years (SD) & $8.67(3.93)$ \\
Mean duration since diagnosis of brain tumor, & $11.87(15.95)$ \\
months (SD) & \\
Diagnosis, N(\%) & $21(67.7 \%)$ \\
Medulloblastoma & $4(12.9 \%)$ \\
Anaplastic ependymoma & $2(6.5 \%)$ \\
Primitive Neuroectodermal Tumor (PNET) & $2(6.5 \%)$ \\
Malignant Glioneuronal Tumor (MGNT) & $2(6.5 \%)$ \\
Atypical Teratoid Rhabdoid Tumor (ATRT) & \\
Residual tumor, N(\%) & $14(45.2 \%)$ \\
Size $<1.5$ cm & $17(54.7 \%)$ \\
Size $>1.5$ cm & \\
Leptomeningeal seeding, N(\%) & $14(45.2 \%)$ \\
No seeding & $17(54.7 \%)$ \\
Seeding & \\
Current stage of applied treatment & $15(48.4)$ \\
Post-CT ,Pre-RT and Pre-PBSCT, N(\%) & $7(22.6)$ \\
Post-CT, Post-RT and Pre-PBSCT, N(\%) & $9(29.0)$ \\
Post-CT, Post-RT and Post-PBSCT, N(\%) & \\
Intelligence (SD) & $95.41(17.60)$ \\
Mean verbal IQ & $78.25(21.69)$ \\
Mean performance IQ & $86.12(17.74)$ \\
Mean full scale IQ & \\
\hline
\end{tabular}

CT: chemotherapy, RT: radiotherapy, PBSCT: Peripheral Blood Stem Cell Transplantation, IQ: intelligence quotient

the summary scores. A 5-point response scale is utilized ( $0=$ never a problem; $1=$ almost never a problem; $2=$ sometimes a problem; $3=$ often a problem; $4=$ almost always a problem). Items are reverse-scored and linearly transformed to a 0 to 100 scale $(0=100,1=75,2=50,3=25,4=0)$, so that higher scores indicate better HRQOL. Scale Scores are computed as the sum of the items divided by the number of items answered.

The ADL measures basic activities of daily living for the 1-Month period prior to interview. Scores of 0 to 2 were assigned to each of 12 items, and higher sum scores of 12 items indicate more difficulties with performing normal self-care activities. For parents of both brain tumor patients and NCs, we administered the parent proxy-report of the PedsQL, the Korean Version of the Parenting Stress Index-Short Form (K-PSI$\mathrm{SF}){ }^{26}$ to assess parenting stress, and the Korean Version of the Parenting Sense of Competence (K-PSOC), ${ }^{26}$ to assess parenting satisfaction and parenting efficacy.

This study protocol was approved by our institutional review board, and written informed consent was obtained from each enrolled participant or, for minors, the participant's parents.

\section{Statistical analysis}

We analyzed the data using SPSS 16.0 R software for Windows. We used the chi-square test, the Mann-Whitney test, and the two-sample t-test to evaluate differences in clinical va- 
riables between the two groups.

Pearson correlation and Spearman's correlation were used to assess the relationship between clinical characteristics with the total scores of PedsQL self- and parent report in brain tumor patients.

The statistical significance level was set at $\mathrm{p}<0.05$.

\section{RESULTS}

There was no significant difference in mean age ( $\mathrm{p}=0.94$, by Mann-Whitney test) or gender ratio ( $\mathrm{p}=0.32$, by Fisher's Exact test) between the brain tumor patients and the NC group.

Table 1 summarizes the clinical characteristics of the children with brain tumors.

Table 2. T-score of $\mathrm{K}-\mathrm{CBCL}$ Subscale in children with brain tumor $(\mathrm{N}=28)$

\begin{tabular}{llc}
\hline & Mean $(\mathrm{SD})$ & Reference value \\
\hline Social subscales & & \\
Total competence & $49.62(9.83)$ & $<33$ \\
Social & $51.62(9.35)$ & $<30$ \\
School & $50.14(10.79)$ & \\
Clinical subscales & & \\
Withdrawn & $55.08(8.80)$ & $>70$ \\
Somatization & $53.96(12.54)$ & \\
Anxious/Depressed & $48.69(7.55)$ & \\
Social problems & $50.19(10.23)$ & \\
Thought problems & $50.35(7.07)$ & \\
Attention problems & $48.15(8.32)$ & \\
Delinquency & $46.46(7.02)$ & \\
Aggression & $46.35(6.12)$ & \\
Internalizing & $51.58(9.95)$ & $>63$ \\
Externalizing & $46.19(6.44)$ & $>63$ \\
Total problem & $48.81(7.69)$ & $>63$ \\
\hline
\end{tabular}

K-CBCL: Korean version of the Child Behavior Checklist
Patients with brain tumors did not show significant behavioral problems or social competent problems in CBCL scale (Table 2).

The mean self-report PedsQL scores on the Physical Health Scale, Emotional Functioning Scale, Social Functioning Scale, School Functioning Scale, and Total Scale were significantly lower in the brain tumor patients than in the NC group. In addition, the mean parent proxy-report PedsQL scores on the Physical Health Scale, Social Functioning Scale, School Functioning Scale, and Total Scale Scores in the brain tumor patients were significantly lower than those of the NC group (Table 3).

There was no significant difference between self-report PedsQL scores and parent proxy-report PedsQL scores in the brain tumor patients (Fig. 1).

The mean ADL score for the brain tumor patients was significantly higher than that of the NC group (Table 4).

Even though there was no significant difference between the two groups in the K-PSI-SF scores of stress related to child learning and in the K-PSOC scores, the K-PSI-SF mean scores related to stress of having a difficult child and to stress of parent-child interaction in the parents of brain tumor patients tended to be higher than those of the NCs' parents (Table 4).

Clinical characteristics including mean age at diagnosis of brain tumor, mean duration since diagnosis of brain tumor, applied treatment and intelligence were not correlated with the total scores of PedsQL self- and parent report in brain tumor patients.

\section{DISCUSSION}

This is the first study measuring HRQOL for children with brain tumors in Korea. In this study, we observed that HRQOL in children with brain tumors was lower than that of normal

Table 3. The PedsQL scores of child self-reports and parent proxy-reports of children with brain tumors and the normal control group

\begin{tabular}{lccc}
\hline & $\begin{array}{c}\text { Children with brain tumor }(\mathrm{N}=31) \\
\text { Mean } \pm \text { SD }\end{array}$ & $\begin{array}{c}\text { Normal control group (N=125) } \\
\text { Mean } \pm \text { SD }\end{array}$ & p \\
\hline Self-report & & & \\
Total score & $58.82(16.83)^{*}$ & $90.68(9.61)$ & 0.00 \\
Physical health & $47.98(26.72)^{*}$ & $93.61(9.93)$ & 0.00 \\
Emotional functioning & $72.06(25.00)^{*}$ & $86.20(16.12)$ & 0.00 \\
Social functioning & $68.24(23.65)^{*}$ & $92.80(15.43)$ & 0.00 \\
School functioning & $52.94(23.66)^{*}$ & $88.40(11.80)$ & \\
Proxy-report & & & 0.00 \\
Total score & $67.78(20.29)^{*}$ & $90.39(10.16)$ & \\
Physical health & $54.60(27.45)^{*}$ & $93.49(12.52)$ & 0.00 \\
Emotional functioning & $82.65(12.00)$ & $86.96(14.59)$ & 0.00 \\
Social functioning & $79.41(26.63)^{*}$ & $93.32(11.18)$ & 0.00 \\
School functioning & $62.35(25.81)^{*}$ & $85.92(14.65)$ & 0.00 \\
\hline
\end{tabular}

${ }^{*} \mathrm{p}<0.01$, by Mann-Whitney test. PedsQL: Pediatric Quality of Life Inventory, version 4.0 


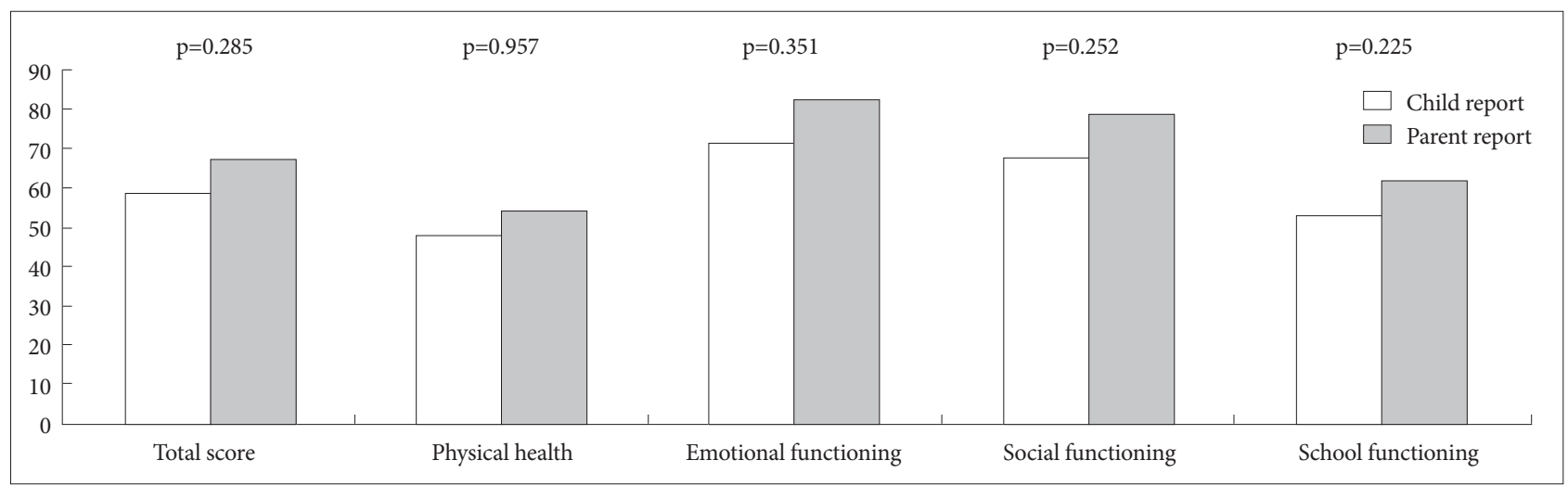

Figure 1. The PedsQL scores between self-report and parent proxy-report of children with brain tumor. PedsQL: Pediatric Quality of Life Inventory, version 4.0 .

Table 4. The ADL, K-PSI-SF, and the K-PSOC scores in the children with brain tumor and normal control subjects

\begin{tabular}{|c|c|c|c|}
\hline & Children with brain tumor $(\mathrm{N}=31)$ & Normal control group $(\mathrm{N}=125)$ & \\
\hline & Mean \pm SD & Mean \pm SD & $P$ \\
\hline $\mathrm{ADL}^{*}$ & $5.12(6.65)^{*}$ & $0.38(0.81)$ & 0.00 \\
\hline \multicolumn{4}{|l|}{ K-PSI-SF } \\
\hline Stress related to difficult child ${ }^{\dagger}$ & $18.23(5.77)^{* *}$ & $15.70(5.72)$ & 0.09 \\
\hline Stress related to parent-child interaction ${ }^{\ddagger}$ & $22.24(4.48)^{* *}$ & $19.93(5.23)$ & 0.09 \\
\hline Stress related to child learning ${ }^{\dagger}$ & $7.18(2.88)$ & $7.20(2.83)$ & 0.84 \\
\hline \multicolumn{4}{|l|}{ K-PSOC } \\
\hline Sense of parenting competence ${ }^{\ddagger}$ & $29.71(4.62)$ & $31.30(5.16)$ & 0.30 \\
\hline Parental anxiety ${ }^{\dagger}$ & $11.71(4.06)$ & $10.87(3.44)$ & 0.36 \\
\hline
\end{tabular}

${ }^{*} \mathrm{p}<0.01,{ }^{* *} \mathrm{p}=0.09$, ${ }^{\text {by }}$ Mann-Whitney test, ${ }^{\prime}$ by Two sample t-test. ADL: activity of daily living, K-PSI-SF: Korean version of the Parenting Stress Index-Short Form, K-PSOC: Korean version of the Parenting Sense of Competence

controls. Our data are generally in keeping with studies of survivors of childhood brain tumor, showing that they had a lower HRQOL than did healthy controls. ${ }^{6,710-12}$ The mean selfreport PedsQL scores, and the mean parent proxy-report PedsQL scores except Emotional Functioning Scale in the brain tumor group were not significantly lower than those of the NC group. However, the mean parent proxy-report PedsQL scores for Emotional Functioning Scale in the brain tumor group were not significantly lower than those of the NC group. Parents are more able to judge a child's external problems but are much less accurate when it comes to judging a child's internal problems. ${ }^{27}$ Therefore, parents are more able to rate a child's HRQOL in relation to physical functioning domains compared to the less visible domains, such emotional functioning. ${ }^{28}$ We demonstrated that children with brain tumors have lower HRQOL, including in the domains of physical health, emotional functioning, social functioning, and school functioning, than health controls have.

In this study, there were no significant differences between the self- and parent-rated HRQOLs in children with brain tumors. Since parents of chronic sick might be able to observe their child accurately, the agreement between parent and child ratings of HRQOL is better in chronically sick than in healthy children. ${ }^{28}$ Although researchers and clinicians should consider pediatric patients' self-reports as the standard for measuring perceived HRQOL, parents' proxy-report is likely needed in some situations when the child is too young, too cognitively impaired, or too ill or fatigued to complete an HRQOL instrument. ${ }^{29}$ Moreover, research has demonstrated the feasibility, reliability, and validity of parent proxy-report for the age subgroup of ages 2-16 years. ${ }^{24}$ Therefore, our findings support the idea that the parent proxy-report substitutes adequately for a patient self-report when childhood patients with brain tumors are not able to complete a self-report, considering that the parent proxy-report underestimates the emotional problem of child. $^{28}$

In this study, we observed that children with brain tumors had more difficulties with performing normal self-care activities compared to healthy controls. Data from the first Childhood Cancer Survivor Study (CCSS) survey indicated that physical performance limitations, including the inability to lift objects, move a table, carry groceries or a bowl, walk uphill, climb a few flights of stairs, bend, lift, stoop, walk one block, eat, dress, bathe, or use the toilet, were prevalent among 19.6\% of childhood cancer survivors. ${ }^{13,25}$ Survivors of childhood brain tumor were most likely to report these performance limi- 
tations, and they were also most likely to report restricted abilities to perform personal care, such as eating, bathing, dressing, or getting around home. ${ }^{13}$ These performance limitations can produce participation restriction, which may place survivors of childhood brain tumor at risk for below optimal attainment of their economic, educational, and occupational goals. ${ }^{25,30}$ Thus, interventions targeted at preventing ADL deterioration and improving ADL in survivors of childhood brain tumor are needed.

In this study, the stress related to having a difficult child and to parent-child interaction in parents of brain tumor patients tended to be higher than those of parents of healthy controls. The stress related to such difficult children includes the child's overall behavioral disturbances, which parents find difficult to handle. In a previous study, parents who reported higher levels of parenting stress reported that their children with cancer had higher levels of acting-out behaviors and emotional problems and fewer positive social behaviors. ${ }^{31}$ The experience of caring for a child with a brain tumor correlates to an increased level of stress that may adversely impact parent mental health, ${ }^{32}$ and this distress persists during ongoing treatments as well as off-treatment periods. ${ }^{33}$ Furthermore, another previous study showed the rate of parent-child dysfunctional interaction was significantly higher in families where a child had a brain tumor than families of healthy controls. ${ }^{34}$ Cancer patients who have good familial interaction have reported less psychological distress and better adjustment. ${ }^{35}$ Thus, further study on more cases is needed to verify that parenting stress in parents of patients with brain tumors is significant higher than that of the parents of health controls.

Though we did not find the relationship of clinical characteristics with the total scores of PedsQL self- and parent report in brain tumor patients, previous studies showed that fatigue, ethnic minority status, male, supratentorial tumors, radiation therapy, and more severe late effects were associated with poorer HRQOL in survivors of pediatric cancers containing brain tumor. ${ }^{410}$ Thus, further study on investigating demographics, disease and treatment factors relating HRQOL in children with brain tumors in Korea is needed.

This study has several limitations. First, as it had a crosssectional design, we did not evaluate HRQOL, ADL, parenting stress, parenting satisfaction, and parenting efficacy for any appreciable duration after the brain tumor diagnosis or after discontinuation of cancer treatment. Therefore, longitudinal studies are needed. Second, we did not evaluate various disease, treatment and demographic factors, including socioeconomic status, educational level, and religion, that may affect HRQOL, ADL, parenting stress, parenting satisfaction, parenting efficacy, and familial interaction. Third, we did not evaluate intelligence function test and $\mathrm{K}-\mathrm{CBCL}$ in the $\mathrm{NC}$ gr- oup, so that we did not compare intelligence, and behavior problems and social competencies between brain tumor patients and NC subjects.

In conclusion, children with brain tumors had lower HRQOL and difficulties in performing ADL. Parents of children with brain tumors had a tendency to experience stress related to having a difficult child and to parent-child interaction. Therefore, we suggest that long-term monitors for the child's HRQOL and ADL, and parenting stress in children with brain tumors are needed.

\section{REFERENCES}

1. Steliarova-Foucher E, Stiller C, Kaatsch P, Berrino F, Coebergh JW, Lacour B, et al. Geographical patterns and time trends of cancer incidence and survival among children and adolescents in Europe since the 1970s (the ACCISproject): an epidemiological study. LANCET 2004;364:2097-2105.

2. Peris-Bonet R, Martinez-Garcia C, Lacour B, Petrovich S, Giner-Ripoll B, Navajas A, et al. Childhood central nervous system tumours--incidence and survival in Europe (1978-1997): report from Automated Childhood Cancer Information System project. Eur J Cancer 2006;42: 2064-2080.

3. Lannering B, Marky I, Lundberg A Olsson E. Long-term sequelae after pediatric brain tumors: their effect on disability and quality of life. Med Pediatr Oncol 1990;18:304-310.

4. Mostow EN, Byrne J, Connelly RR, Mulvihill JJ. Quality of life in longterm survivors of CNS tumors of childhood and adolescence. J Clin Oncol 1991;9:592-599.

5. Zebrack BJ, Gurney JG, Oeffinger K, Whitton J, Packer RJ, Mertens A, et al. Psychological outcomes in long-term survivors of childhood brain cancer: a report from the childhood cancer survivor study. J Clin Oncol 2004;22:999-1006.

6. Barr RD, Simpson T, Whitton A, Rush B, Furlong W, Feeny DH. Health-related quality of life in survivors of tumours of the central nervous system in childhood--a preference-based approach to measurement in a cross-sectional study. Eur J Cancer 1999;35:248-255.

7. Cardarelli C, Cereda C, Masiero L, Viscardi E, Faggin R, Laverda A, et al. Evaluation of health status and health-related quality of life in a cohort of Italian children following treatment for a primary brain tumor. Pediatr Blood Cancer 2006;46:637-644.

8. Eiser C, Vance YH, Horne B, Glaser A, Galvin H. The value of the PedsQLTM in assessing quality of life in survivors of childhood cancer. Child Care Health Dev 2003;29:95-102.

9. Fluchel M, Horsman JR, Furlong W, Castillo L, Alfonz Y, Barr RD. Self and proxy-reported health status and health-related quality of life in survivors of childhood cancer in Uruguay. Pediatr Blood Cancer 2008; 50:838-843.

10. Meeske KA, Patel SK, Palmer SN, Nelson MB, Parow AM. Factors associated with health-related quality of life in pediatric cancer survivors. Pediatr Blood Cancer 2007;49:298-305.

11. Odame I, Duckworth J, Talsma D, Beaumont L, Furlong W, Webber C, et al. Osteopenia, physical activity and health-related quality of life in survivors of brain tumors treated in childhood. Pediatr Blood Cancer 2006;46:357-362.

12. Speechley KN, Barrera M, Shaw AK, Morrison HI, Maunsell E. Healthrelated quality of life among child and adolescent survivors of childhood cancer. J Clin Oncol 2006;24:2536-2543.

13. Ness KK, Mertens AC, Hudson MM, Wall MM, Leisenring WM, Oeffinger $\mathrm{KC}$, et al. Limitations on physical performance and daily activities among long-term survivors of childhood cancer. Ann Intern Med 2005; 143:639-647. 
14. Fuemmeler BF, Mullins LL, Marx BP. Posttraumatic stress and general distress among parents of children surviving a brain tumor. Child Health Care 2001;30:169-182.

15. Chien LY, Lo LH, Chen CJ, Chen YC, Chiang CC, Yu Chao YM. Quality of life among primary caregivers of Taiwanese children with brain tumor. Cancer Nurs 2003;26:305-311.

16. Wijnberg-Williams BJ, Kamps WA, Klip EC, Hoekstra-Weebers JE. Psychological adjustment of parents of pediatric cancer patients revisited: five years later. Psychooncology 2006;15:1-8.

17. World Health Organization. Constitution of the World Health Organization: Basic Document. Geneva, Switzerland: World Health Organization; 1948.

18. Varni JW, Burwinkle TM, Lane MM. Health-related quality of life measurement in pediatric clinical practice: an appraisal and precept for future research and application. Health Qual Life Outcomes 2005;3:34.

19. Matza LS, Swensen AR, Flood EM, Secnik K, Leidy NK. Assessment of health-related quality of life in children: a review of conceptual, methodological, and regulatory issues. Value Health 2004;7:79-92.

20. Schell LM. Culture as a stressor: a revised model of biocultural interaction. Am J Phys Anthropol 1997;102:67-77.

21. Park HW, Kwak KJ, Park KB. The development of Korean version of WPPSI : The standardization study (1). Korean J Dev Psychol 1996;9:6070.

22. Kwak KJ, Park HW, Kim CT. A Study for the standardization of Korean WISC-3 (1). Korean J Dev Psychol 2002;15:19-33.

23. Han MH, Yoo AJ. The Validation of the child behavior checklist. Korean J Child Stud 1995;16:5-21.

24. Varni JW, Limbers CA, Burwinkle TM. Parent proxy-report of their children's health-related quality of life: an analysis of 13,878 parents' reliability and validity across age subgroups using the PedsQL 4.0 Generic Core Scales. Health Qual Life Outcomes 2007;5:2.

25. Ness KK, Hudson MM, Ginsberg JP, Nagarajan R, Kaste SC, Marina N, et al. Physical performance limitations in the Childhood Cancer Survivor Study cohort. J Clin Oncol 2009;27:2382-2389.
26. Shin SJ, Chung MJ. Effects of stress, social support and efficacy on mothers' parenting behaviors. Korean J Child Stud 1998;19:27-42.

27. Achenbach TM, McConaughy SH, Howell CT. Child/adolescent behavioral and emotional problems: implications of cross-informant correlations for situational specificity. Psychol Bull 1987;101:213-232.

28. Eiser C, Morse R. Can parents rate their child's health-related quality of life? Results of a systematic review. Qual Life Res 2001;10:347-357.

29. Hays RM, Valentine J, Haynes G, Geyer JR, Villareale N, McKinstry B, et al. The Seattle Pediatric Palliative Care Project: effects on family satisfaction and health-related quality of life. J Palliat Med 2006;9:716-728.

30. Ness KK, Gurney JG, Zeltzer LK, Leisenring W, Mulrooney DA, Nathan $\mathrm{PC}$, et al. The impact of limitations in physical, executive, and emotional function on health-related quality of life among adult survivors of childhood cancer: a report from the Childhood Cancer Survivor Study. Arch Phys Med Rehabil 2008;89:128-136.

31. Colletti CJ, Wolfe-Christensen C, Carpentier MY, Page MC, McNallKnapp RY, Meyer WH, et al. The relationship of parental overprotection, perceived vulnerability, and parenting stress to behavioral, emotional, and social adjustment in children with cancer. Pediatr Blood Cancer 2008;51:269-274.

32. Witt WP, Litzelman K, Wisk LE, Spear HA, Catrine K, Levin N, et al. Stress-mediated quality of life outcomes in parents of childhood cancer and brain tumor survivors: a case-control study. Qual Life Res 2010;19: 995-1005.

33. Hutchinson KC, Willard VW, Hardy KK, Bonner MJ. Adjustment of caregivers of pediatric patients with brain tumors: a cross-sectional analysis. Psychooncology 2009;18:515-523.

34. Radcliffe J, Bennett D, Kazak AE, Foley B, Phillips PC. Adjustment in childhood brain tumor survival: child, mother, and teacher report. J Pediatr Psychol 1996;21:529-539.

35. Badr H, Taylor CL. Effects of relationship maintenance on psychological distress and dyadic adjustment among couples coping with lung cancer. Health Psychol 2008;27:616-627. 